\title{
Fluvastatin improves osteoporosis in fructose-fed insulin resistant model rats through blockade of the classical mevalonate pathway and antioxidant action
}

\author{
RIE HANAYAMA ${ }^{1,2}$, HIDEO SHIMIZU ${ }^{1,2}$, HIRONORI NAKAGAMI ${ }^{3}$, MARIANA KIOMY OSAKO ${ }^{1}$, \\ HIROFUMI MAKINO ${ }^{1,2}$, YASUO KUNUGIZA ${ }^{4}$, TETSUYA TOMITA ${ }^{4}$, IKUYO TSUKAMOTO ${ }^{5}$, \\ HIDEKI YOSHIKAWA ${ }^{4}$, HIROMI RAKUGI ${ }^{2}$ and RYUCHI MORISHITA ${ }^{1}$

\begin{abstract}
Division of ${ }^{1}$ Clinical Gene Therapy, ${ }^{2}$ Department of Geriatric Medicine, ${ }^{3}$ Division of Gene Therapy Science,
${ }^{4}$ Department of Orthopaedic Surgery, Osaka University, Graduate School of Medicine, 2-2 Yamadaoka, Suita,
\end{abstract} \\ Osaka 565-0871; ${ }^{5}$ Department of Food Science and Nutrition, Nara Women's University, Nara 630-8506, Japan
}

Received October 22, 2008; Accepted December 8, 2008

DOI: 10.3892/ijmm_00000167

\begin{abstract}
Feeding rats with a high-fructose diet induced insulin resistance, leading to hypertension or metabolic disorders. Although hypertension is known to accelerate osteoporosis, it is not obvious whether insulin resistance would accelerate osteoporosis. In this study, we evaluated whether osteoporosis might accelerate in fructose-fed rats (FFR), and examined the effect of fluvastatin through a blockade of the mevalonate pathway and an antioxidant action. Stimulation of recombinant receptor activator of nuclear factor-kappaB (NF-кB) ligand (RANKL) expressed by osteoblasts/ stromal cells and macrophage-colony stimulating factor (M-CSF) significantly increased TRAP-positive multinuclear osteoclasts and pit formation, accompanied by an increase in reactive oxygen species as assessed by dichlorodihydrofluorescein (DCF) staining. Interestingly, it was completely abolished by treatment with fluvastatin, pyrrolidine dithiocarbamate (PDTC) and N-acetylcysteine (NAC), but not pravastatin. These actions of fluvastatin were partially abolished by cotreatment with geranylgeranylpyrophosphate (GGPP), but not farnesylpyrophosphate (FPP). In the estrogen-deficient model by ovariectomy, FFR exhibited a decrease in bone mineral density, activation of osteoclasts, and an increase in urinary deoxypyridinoline. Importantly, the treatment of fluvastatin, but not pravastatin, attenuated FFR-induced osteoporosis. The present study demonstrates that fructose fed to rats induced insulin resistance and accelerated osteoporosis, while fluvastatin, but not pravastatin, significantly attenuated osteo-
\end{abstract}

Correspondence to: Professor Ryuichi Morishita, Division of Clinical Gene Therapy, Osaka University, Graduate School of Medicine, 2-2 Yamadaoka, Suita, Osaka 565-0871, Japan E-mail: morishit@cgt.med.osaka-u.ac.jp

Key words: osteoclast, osteoporosis, antioxidant, statins clast differentiation and activation through a blockade of the classical mevalonate pathway and an antioxidant action, leading to prevention of osteoporosis.

\section{Introduction}

Diabetic osteoporosis is increasingly recognized as a significant comorbidity of type I diabetes mellitus (DM), but not type II DM in the clinical view point $(1,2)$. Thus, the analysis of type I DM may serve as a more straightforward system to understand the effect of suppressed insulin signaling, hyperglycemia, and metabolic abnormalities on regulation of bone formation. However, endocrine problems, such as, thyrotoxicosis or primary hyperparathyroidism, may cause osteoporosis in individuals with type I DM (3). Thus, it might be difficult to understand 'diabetic osteoporosis'.

There is also interest in whether pharmacotherapy used to lower blood lipids, 3-hydroxyl-3-methylglutaryl coenzyme A (HMG-CoA) reductase inhibitors (statins), may increase bone mineral density. In 1999, experimental work produced the novel finding that statins have direct effects on bone formation through induction of bone morphogenetic protein-2 (BMP-2) in osteoblasts. In a cross-sectional population study, bone mineral density was approximately $7-8 \%$ higher in women taking statins than in controls (4), and a case control study showed that current use of statins is associated with a reduced risk of fracture $(5,6)$. However, two large studies failed to demonstrate an association between statins and risk of fracture. In the LIPID study, there was no difference in fracture occurrence in the pravastatin group compared to the placebo group (7). In another study using General Practice database, no association was found between the use of statins and risk of fracture in 81,880 individuals (8). From these results, we speculate that individual statins have different effects on bone metabolism.

High blood pressure is associated with abnormalities of calcium metabolism, leading to an increase in calcium loss, secondary activation of the parathyroid gland, and increased movement of calcium from bone, thereby increasing the risk 
of osteoporosis $(9,10)$. However, it is not understood whether insulin resistance or metabolic syndrome is associated with osteoporosis. A previous report suggests that increased dietary fructose in rodents recapitulates many aspects of metabolic syndrome with hypertension, insulin resistance and dyslipidemia (11). Thus, in this study, we evaluated whether osteoporosis would be accelerated in the fructose-fed rats (FFR), and examined the effect of statins on FFR-induced osteoporosis. In addition, we examined the effects of fluvastatin on osteoclasts, especially focusing on its antioxidant action, since fluvastatin has a scavenging effect on hydroxyl radicals due to its unique chemical structure (12). We demonstrated for the first time that fructose-feeding accelerated osteoporosis, while fluvastatin, but not pravastatin, attenuated osteoporosis and inhibited osteoclast differentiation and activation through both an antioxidant effect and inhibition of HMG-CoA reductase.

\section{Materials and methods}

Rat ovariectomy osteoporosis model. Female adult Wistar rats (10 weeks old) were purchased from SLC Japan (Shizuoka, Japan). After the rats were anesthetized with intraperitoneal ketamine $(80 \mathrm{mg} / \mathrm{kg})$ and xylazine $(10 \mathrm{mg} / \mathrm{kg})$, bilateral ovariectomy was performed. Rats were fed fructoserich chow containing $60 \%$ fructose, $5 \%$ fat, and $20 \%$ protein (Oriental Yeast Co.) for 28 days. In some groups of rats, fluvastatin or pravastatin $(5 \mathrm{mg} / \mathrm{kg} /$ day $)$ was orally administered every day. Four weeks after the operation, systolic blood pressure was measured using the tail-cuff method (BP98A, Softron), and they were deeply anesthetized and sacrificed to collect the femurs, tibias, and blood for biochemical analysis. Osteoclasts in trabecular bone under growth plate in proximal tibia were analyzed by light microscope and tartrate-resistant acid phosphatase (TRAP) stained area per field $(300 \mu \mathrm{m} \times 300 \mu \mathrm{m})$ was measured by Image $\mathbf{J}$ software, NIH.

Dual energy X-ray absorptiometry (DEXA) and microcomputed tomography. Bone density measurements were performed by dual-photon X-ray absorptiometry (DEXA) bone densitometry (GE-Lunar DPX-IQ, Madison, WI). Highand low-beam energies for all scans were $80 \mathrm{kV}$ and $35 \mathrm{kV}$, respectively, at $0.5 \mathrm{~mA}$ as previously described (13). Bone mineral density (BMD) was obtained in $\mathrm{g} / \mathrm{cm}^{2}$.

Materials and cell culture. Pravastatin was obtained from Wako (Osaka, Japan) and fluvastatin was obtained from Novartis. Other materials were obtained from Sigma Chemical Co., (St. Louis, MO).

Bone marrow cells were obtained from 3-day-old neonatal white rabbits as previously described (14). Briefly, rabbit bone marrow cells were flushed out from the femurs and tibiae, collected into tubes, and washed twice with PBS. The mononuclear-rich cell fraction was separated from marrow cells and cultured ( $1 \times 10^{5}$ cells/well of 24 -well plate) in $\alpha$-MEM medium containing $10 \%$ fetal bovine serum.

Osteoclast differentiation was also examined using rat osteoclast culture system obtained from Primary Cell Company (Primary Cell Co., Ltd., Sapporo). Rat osteoclast precursor cells seeded in a 24-well plate were incubated with macrophagecolony stimulating factor (M-CSF: $50 \mathrm{ng} / \mathrm{ml}$ ) and receptor activator of nuclear factor-kappaB (NF-кB) ligand (RANKL: $50 \mathrm{ng} / \mathrm{ml}$ ) expressed by osteoblasts/stromal cells containing medium with or without several stimulants for 5 days to examine the differentiation of osteoclasts. Cell viability of osteoclasts was measured using MTS [3-(4,5-dimethylthiazol2-yl)-5-(3-carboxymethoxyphenyl)-2-(4-sulfophenyl)-2Htetrazolium] assay after $48 \mathrm{~h}$ of treatment with several stimulants. Approximately $100 \mu 1$ CellTiter 96 One Solution Reagent (Promega, Madison, WI) in $500 \mu 1$ Dulbecco's modified Eagle's medium (DMEM) was added to each well, and absorbance was measured at $490 \mathrm{~nm}$.

ROS (reactive oxygen species) production by dichlorodihydrofluorescein (DCF) staining. To measure intracellular ROS production, rat osteoclasts were treated with M-CSF $(50 \mathrm{ng} / \mathrm{ml})$ and RANKL $(50 \mathrm{ng} / \mathrm{ml})$ and/or several stimulants for $24 \mathrm{~h}$, and loaded with $10 \mu \mathrm{mol} / \mathrm{l} \mathrm{CM}$-DCFH-DA for $15 \mathrm{~min}$ at room temperature in the dark. During loading, the acetate groups on CM-DCFH-DA are moved by intracellular esterase, trapping the probe inside the cells. Although several dihydrofluorescein derivatives were used to measure intracellular ROS generation $(15,16)$, CM-DCFH-DA was chosen because it exhibits better retention in cells than other derivatives (17). The production of ROS was measured by fluorescence under fluorescence microscopy.

Tartrate-resistant acid phosphatase (TRAP) staining. After treatment with vitamin D3 $\left(1 \times 10^{-8} \mathrm{M}\right)$ and several stimulants for 5 days, mononuclear cells were fixed with $4.0 \%$ paraformaldehyde in PBS for $10 \mathrm{~min}$ at room temperature before being stained for TRAP. Rat osteoclasts were treated similarly after treatment with M-CSF (50 ng/ml) and RANKL (50 ng/ml) and several stimulants. Enzyme histochemical staining for TRAP was performed as previously reported (18).

Pit formation. Osteoclast activation was evaluated by the inhibitory effect on RANKL-induced pit-forming activity, as previously reported (19). We used osteologic calcium hydroxyapatite-coated slides (BD Biocoat; BD Biosciences, Bedford, MA). Rat osteoclasts $\left(1 \times 10^{5}\right.$ cells/well $)$ were incubated with RANKL $(50 \mathrm{ng} / \mathrm{ml})$ and M-CSF $(50 \mathrm{ng} / \mathrm{ml})$ with several stimulants for 7 days. On day 8 , cells were washed vigorously and the area of resorption of calcified matrix on each disc was measured.

Statistical analysis. All values are expressed as mean \pm SEM. Analysis of variance with subsequent Bonferroni's/Dunnet's test was employed to determine the significance of differences in multiple comparisons. Values of $\mathrm{P}<0.05$ were considered statistically significant.

\section{Results}

Effects of statins on rat osteoclasts. To examine the effects of fluvastatin and pravastatin, we employed rat osteoclast culture. Treatment with M-CSF (50 ng/ml) and RANKL (50 ng/ml) induced osteoclast differentiation as assessed by TRAP staining, whereas co-treatment with fluvastatin $(100 \mathrm{nM})$, but 


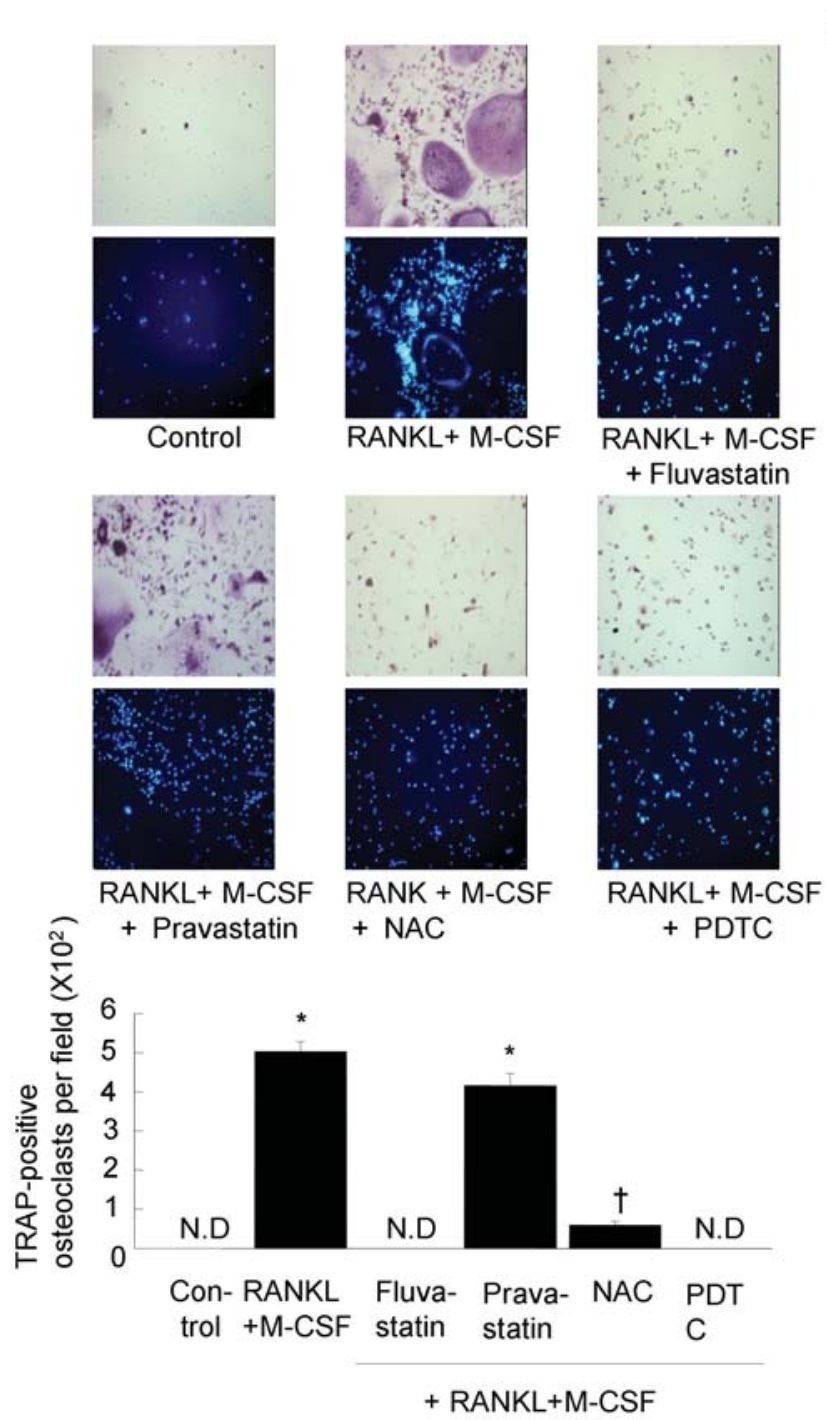

Figure 1. Effects of fluvastatin, pravastatin, PDTC and NAC on rat osteoclast differentiation and activation. (A) Representative photomicrographs and number of TRAP-positive staining cells. (B) Pit formation assay (C) DCF staining. Control, no treatment; RANKL+M-CSF, RANKL (50 ng/ml) and M-CSF $(50 \mathrm{ng} / \mathrm{ml})$. Under treatment with RANKL and M-CSF, fluvastatin $(100 \mathrm{nM})$, pravastatin $(100 \mathrm{nM})$, PDTC $(10 \mu \mathrm{M})$ and NAC $(10 \mathrm{mM})$ were administered. ${ }^{*} \mathrm{P}<0.01$ vs. control; ${ }^{\dagger} \mathrm{P}<0.01$ vs. RANKL+M-CSF. N.D, not detected. $n=4-8$ per group.

not pravastatin $(100 \mathrm{nM})$, significantly attenuated the number of TRAP-positive multinuclear cells induced by M-CSF and RANKL ( $<<0.01$, Fig. 1A). To elucidate the differential effects of statins on the number of TRAP-positive cells, we focused on the role of oxidative stress. Importantly, a potent antioxidant, pyrrolidine dithiocarbamate (PDTC: $10 \mu \mathrm{M}$ ) as well as $\mathrm{N}$-acetylcysteine (NAC: $10 \mathrm{mM}$ ), significantly decreased the number of TRAP-positive multinuclear cells induced by M-CSF and RANKL ( $\mathrm{P}<0.01$, Fig. 1A). To examine further functional aspects, we employed the pit formation assay, whose results are well correlated with the function of TRAP-positive cells in rat osteoclasts under treatment with M-CSF and RANKL. Similarly, treatment with fluvastatin, PDTC or NAC significantly attenuated the area of pit formation on an osteologic field induced by MCSF and RANKL, while pravastatin did not (Fig. 1B). To
A

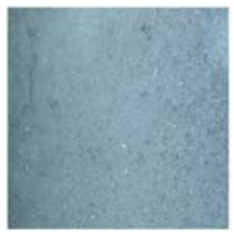

Control

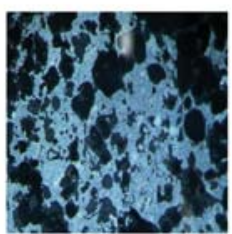

RANKL+M-CSF

+ Pravastatin
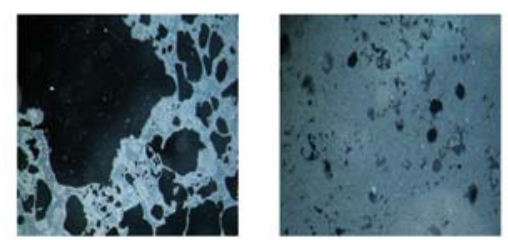

RANKL + M-CSF RANKL +M-CSF + Fluvastatin

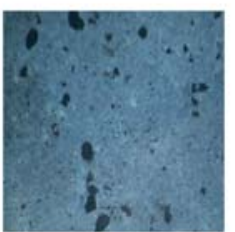

$\mathrm{RANKL}+\mathrm{M}-\mathrm{CSF}$ + NAC

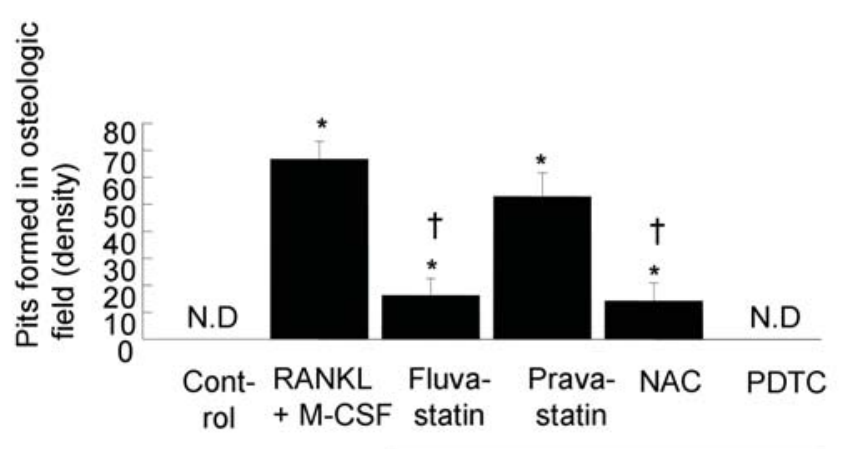

+RANKL + M-CSF

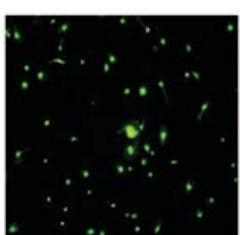

Control

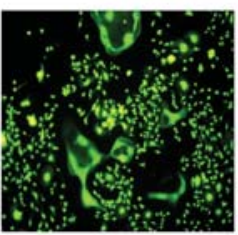

RANKL+ M- CSF + Pravastatin

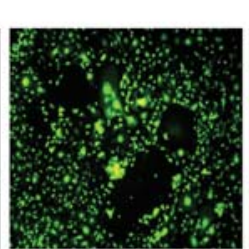

RANKL +M-CSF RANKL +M-CSF
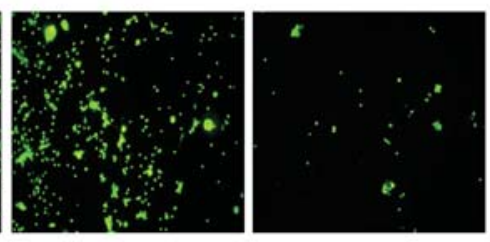

RANKL+M-CSF RANKL+M-CSF + NAC + PDTC

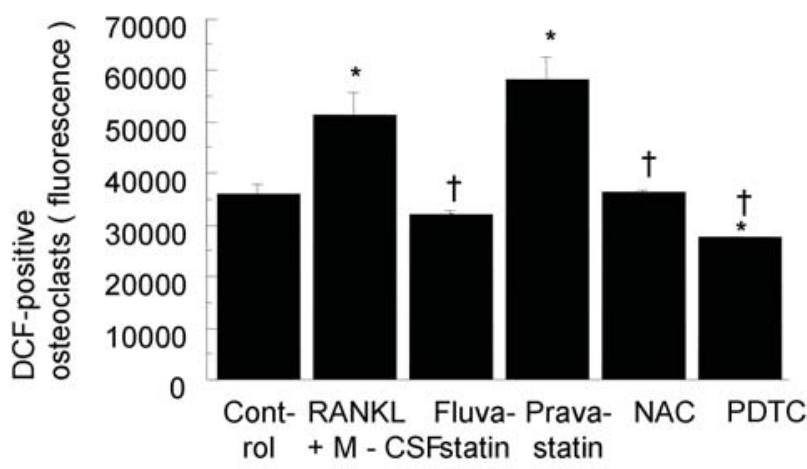

+ RANKL+M-CSF 
A

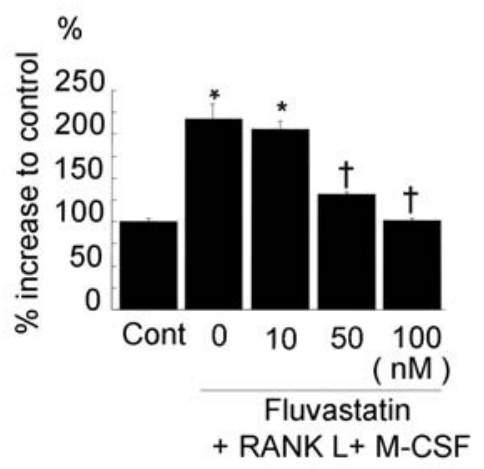

C $\%$

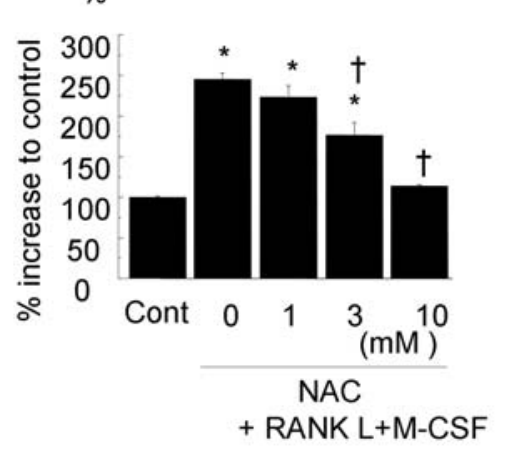

D
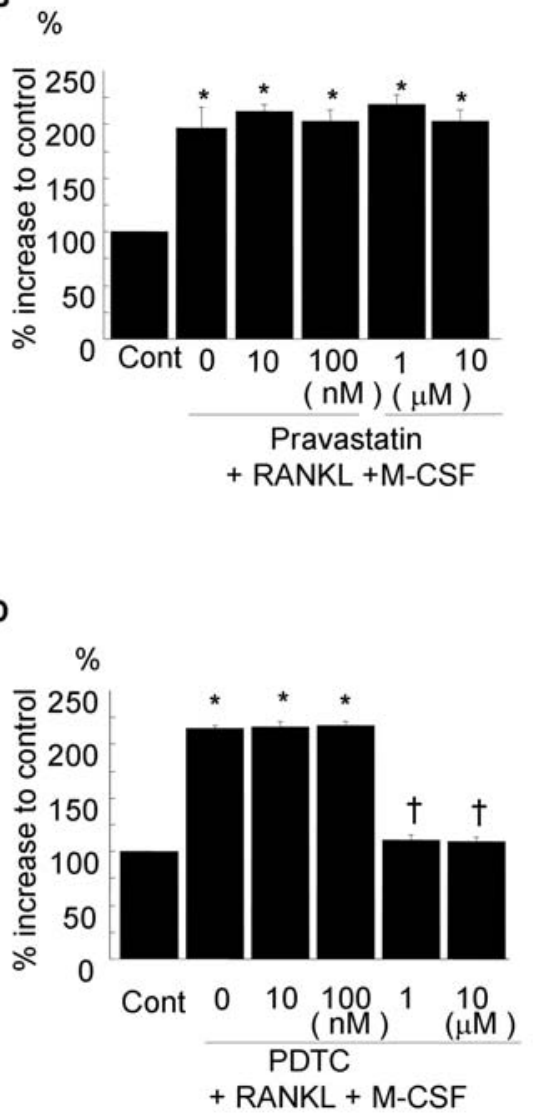

Figure 2. Effect of fluvastatin, pravastatin and PDTC on cell growth as assessed by MTS assay in a dose-dependent manner. Control, no treatment; RANKL+M-CSF, RANKL $(50 \mathrm{ng} / \mathrm{ml})$ and M-CSF $(50 \mathrm{ng} / \mathrm{ml})$. Under treatment with RANKL and M-CSF, fluvastatin, pravastatin, PDTC and NAC were administered in several doses. ${ }^{*} \mathrm{P}<0.01$ vs. control; ${ }^{\dagger} \mathrm{P}<0.01$ vs. RANKL+M-CSF. $\mathrm{n}=4-8$ per group.

clarify whether the inhibitory effects of fluvastatin might be due to antioxidant effects, we measured ROS production as assessed by DCF staining. As shown in Fig. 1C, treatment with M-CSF and RANKL significantly induced ROS production, whereas co-treatment with fluvastatin, PDTC or NAC completely inhibited ROS production. In contrast, pravastatin did not decrease ROS production.

Since the number of TRAP-positive cells correlated with cell viability, assessed by MTS assay in rat osteoclasts, we examined cell viability under treatment with M-CSF and RANKL. As shown in Fig. 2, co-treatment with fluvastatin or NAC, but not pravastatin, significantly attenuated an increase in cell viability induced by treatment with M-CSF and RANKL in a dose-dependent manner. Treatment with PDTC completely blocked it even at a low concentration $(1 \mu \mathrm{M})$.

It is well known that statins reduce the synthesis of other isoprenoid intermediates in the mevalonate pathway, such as farnesylpyrophosphate (FPP) and geranylgeranylpyrophosphate (GGPP), and the prenylation of small GTP-binding protein $(20,21)$, which block osteoclast activity and inhibit osteoblast apoptosis. Thus, we examined the effects of these isoprenoid intermediates in rat osteoclasts. The inhibitory effects of fluvastatin on osteoclast differentiation and activation assessed by the number of TRAP-positive cells and pit formation, were partially abolished by co-treatment with GGPP, but not FPP (Fig. 3A and B). Moreover, co-treatment with GGPP, but not
FPP, partially attenuated antioxidant effects of fluvastatin as assessed by DCF staining (Fig. 3C).

Effect of fluvastatin on rabbit mononuclear cells. We also examined the effects of fluvastatin in rabbit mononuclear cells which may include pre-osteoblasts and pre-osteoclasts. Treatment with vitamin D3 $\left(1 \times 10^{-8} \mathrm{~mol} / \mathrm{l}\right)$ significantly increased the number of TRAP-positive mononuclear cells, whereas treatment with fluvastatin, PDTC or NAC abolished them (Fig. 4A). In addition, the inhibitory effect of fluvastatin on osteoclast differentiation assessed by the number of TRAP-positive cells, was only partially abolished by cotreatment with GGPP, but not FPP (Fig. 4B). These results suggest that the inhibition of osteoclast differentiation and activation by treatment with fluvastatin might be due to both an antioxidant effect and the loss of GGPP.

Effect of statins on osteoporosis of fructose-fed rats. Finally, we exminaed the effects of fluvastatin and pravastatin in in vivo osteoporosis model. Initially, to clarify whether fructose-fed insulin resistance would accelerate osteoporosis, we employed rat ovariectomy model of estrogen deficiency as a model of osteoporosis with fructose-fed rat. We previously confirmed that at 28 days after bilateral ovariectomy, serum estradiol level was significantly decreased in the ovariectomy group, while there was no significant difference in body weight (21). 


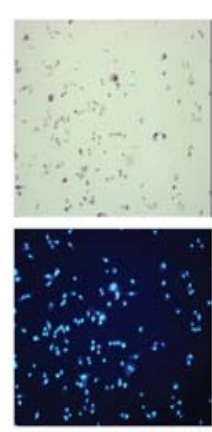

RANKL+M-CSF + Fluvastatin

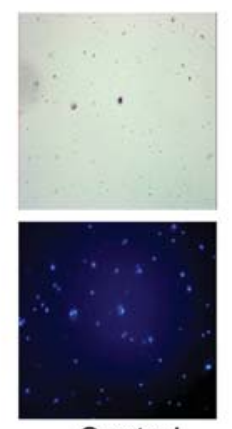

Control

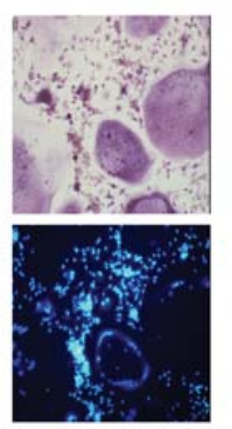

RANKL+M-CSF
A
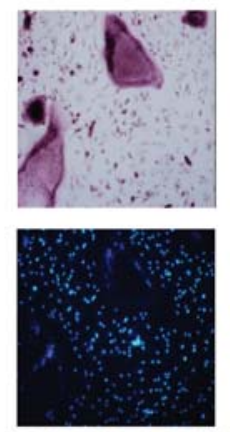

RANKL+M-CSF

+ Fluvastatin

$+\operatorname{GGPP}(5 \mu \mathrm{M})$

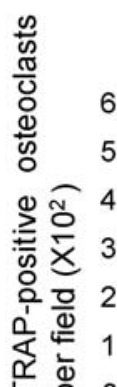

๕

$+\operatorname{FPP}(5 \mu \mathrm{M})$
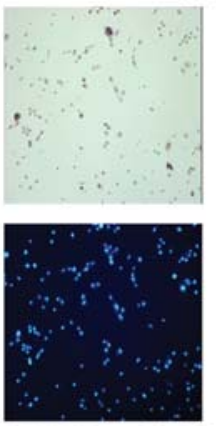

RANKL+M-CSF

+ Fluvastatin $P<0.01$

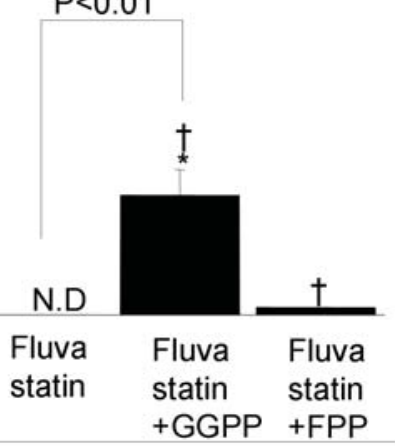

+ RANKL+M-CSF
Figure 3. Effect of GGPP and FPP on TRAP-positive cells. (A) Representative photomicrographs and number of TRAP-positive staining cells in rat osteoclasts. (B) Pit formation assay. (C) DCF staining. Control, no treatment; RANKL+M-CSF: RANKL $(50 \mathrm{ng} / \mathrm{ml})$ and M-CSF $(50 \mathrm{ng} / \mathrm{ml})$. Under treatment with RANKL and M-CSF, fluvastatin $(100 \mathrm{nM})$ was administered with/without the addition of farnesylpyrophosphate (FPP, $5 \mu \mathrm{M}$ ) or geranylgeranylpyrophosphate (GGPP, $5 \mu \mathrm{M})$. ${ }^{*} \mathrm{P}<0.01$ vs. control; ${ }^{\dagger} \mathrm{P}<0.01$ vs RANKL+M-CSF. N.D, not detected. $n=4-8$ per group.

Systolic blood pressure and blood sugar were significantly increased in fructose-fed rat (no treatment: SBP $110 \pm 2 \mathrm{mmHg}$ and $\mathrm{BS} 127 \pm 5 \mathrm{mg} / \mathrm{dl}$; fructose-fed $119 \pm 3 \mathrm{mmHg}$ and $\mathrm{BS}$ $144 \pm 5 \mathrm{mg} / \mathrm{dl}, \mathrm{P}<0.01$ ), as shown in Table I. Osteoclast activity, assessed by quantification of TRAP-positive stained area, was significantly increased in the tibia of ovariectomized rats with fructose-feeding (Fig. 5A). Bone density, assessed by dual-energy X-ray absorptiometry, was also significantly decreased in the tibia of ovariectomized rats with fructosefeeding (Fig. 5B). These results were accompanied by a significant change in urinary deoxypyridinoline, which is released from bone by the processing of tissue collagen.

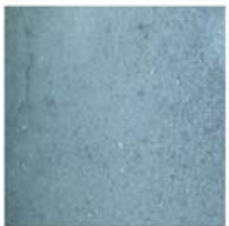

Control

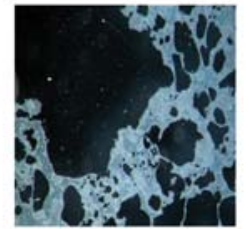

RANKL+M-CSF

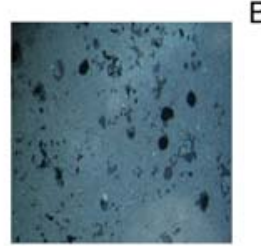

RANKL+M-CSF + Fluvastatin
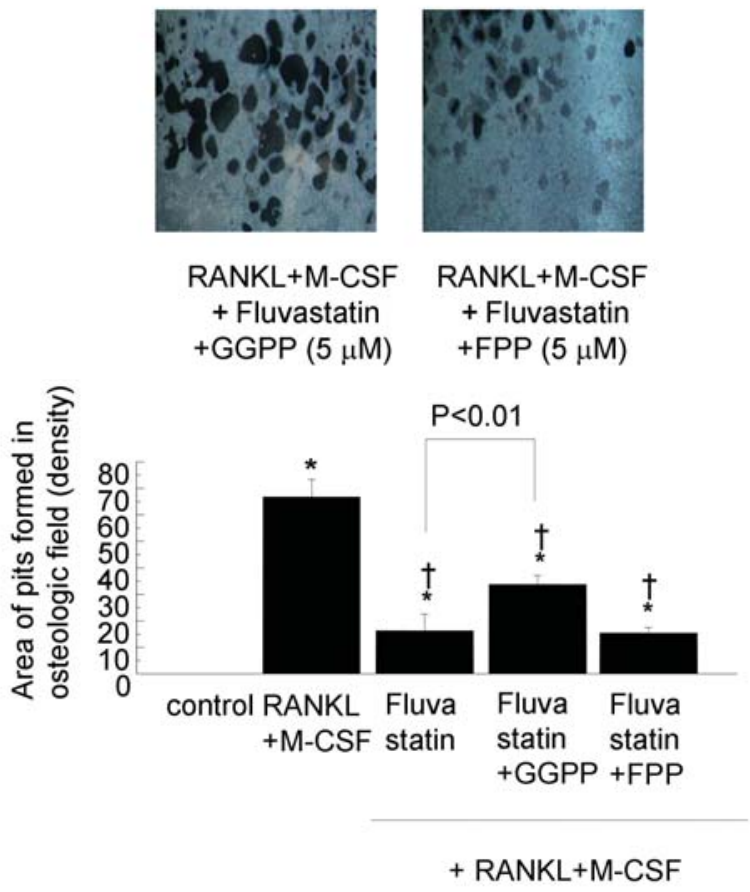

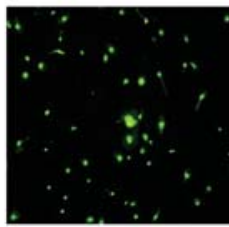

Control

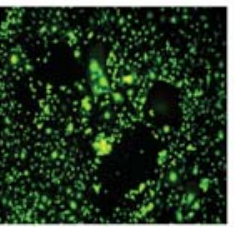

RANKL+M-CSF RANKL+M-CSF + Fluvastatin

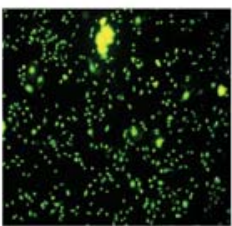

RANKL+M-CSF

+ Fluvastatin

+ GGPP $5 \mu \mathrm{M}$

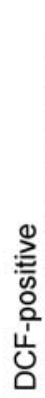

70000

60000 50000

40000

30000

20000 10000

0

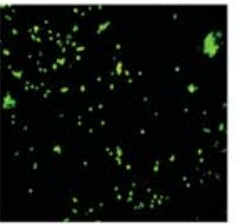

RANKL+M-CSF

+ Fluvastatin

+ FPP $5 \mu \mathrm{M}$

$P<0.01$
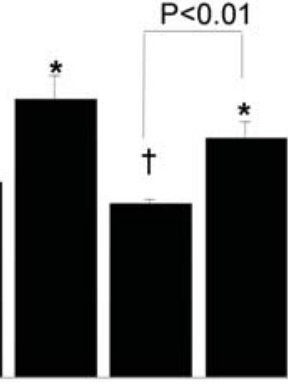

$\dagger$

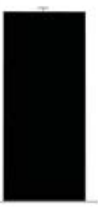

Cont- RANKL Fluva- Fluva Fluva rol + M-CSF Statin statin statin +GGPP +FPP 


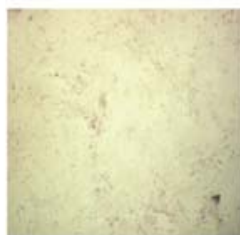

Control

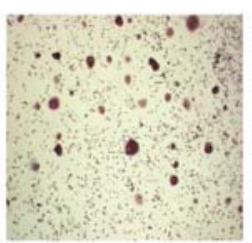

VitaminD3

+ Pravastatin

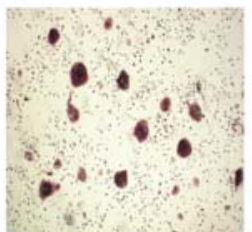

VitaminD3

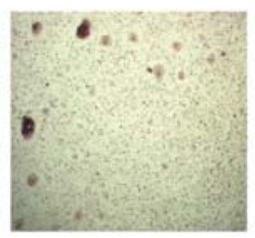

VitaminD3

+ NAC

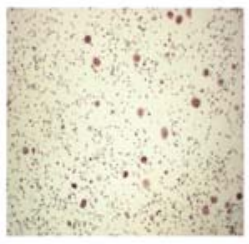

VitaminD3

+ Fluvastatin

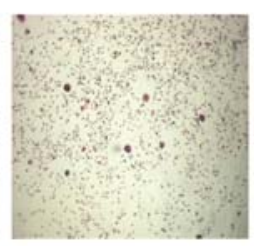

VitaminD3 + PDTC
A
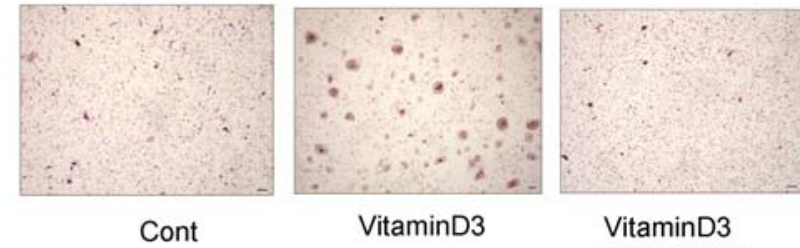

VitaminD3

+ Fluvastatin
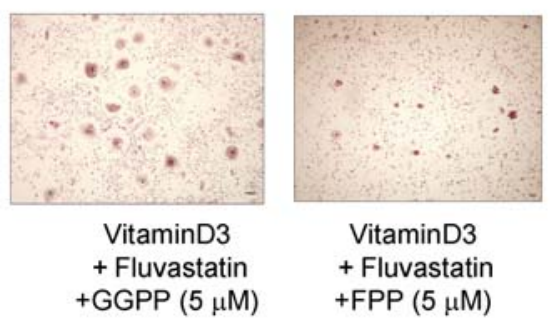

VitaminD3

+ Fluvastatin

$+\mathrm{FPP}(5 \mu \mathrm{M})$

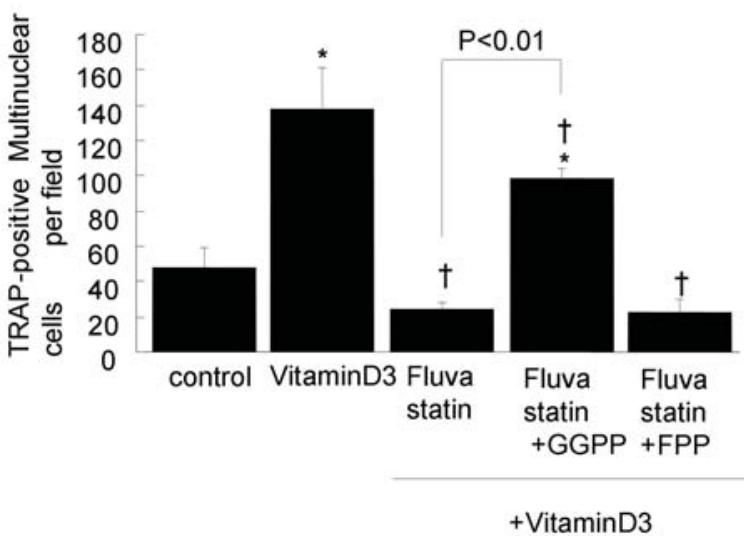

Figure 4. Effects of fluvastatin, pravastatin, PDTC and NAC on osteoclast differentiation in rabbit mononuclear cells. (A and B) Representative photomicrographs and numbers of TRAP-positive staining cells in rabbit mononuclear cells. Control, no treatment; RANKL+M-CSF: RANKL (50 ng/ml) and M-CSF $(50 \mathrm{ng} / \mathrm{ml})$. Under treatment with RANKL and M-CSF, fluvastatin $(100 \mathrm{nM})$, pravastatin $(100 \mathrm{nM})$, PDTC $(10 \mu \mathrm{M})$ and NAC (10 mM) were administered. Under treatment with RANKL and M-CSF, fluvastatin (100 nM) was administered with/without farnesylpyrophosphate (FPP: $5 \mu \mathrm{M})$ or geranylgeranylpyrophosphate (GGPP: $5 \mu \mathrm{M}$ ). ${ }^{*} \mathrm{P}<0.01$ vs. Control; ${ }^{\dagger} \mathrm{P}<0.01$ vs. RANKL+M-CSF. N.D, not detected; $\mathrm{n}=4-8$ per group.

Table I. Body weight, blood pressure and serum cholesterol levels in FFR-induced rat with or without the treatment of fluvastatin.

\begin{tabular}{lcccc}
\hline & Wild+ovx & FFR+ovx & $\begin{array}{c}\text { FFR+ovx } \\
+ \text { Fluvastatin }\end{array}$ & $\begin{array}{c}\text { FFR+ovx } \\
+ \text { Pravastatin }\end{array}$ \\
\hline Body weight (g) & $301 \pm 4$ & $297 \pm 3$ & $287 \pm 3$ & $298 \pm 6$ \\
SBP (mmHg) & $110 \pm 2$ & $119 \pm 3^{\text {a }}$ & $116 \pm 2$ & $113 \pm 3$ \\
DBP (mmHg) & $82 \pm 2$ & $86 \pm 2$ & $83 \pm 1$ & $83 \pm 2$ \\
Glucose (mg/dl) & $127 \pm 5$ & $144 \pm 5^{\text {a }}$ & $132 \pm 5$ & $147 \pm 6^{\text {a }}$ \\
Insulin (ng/ml) & $0.2 \pm 0.02$ & $0.4 \pm 0.03^{\text {a }}$ & $0.3 \pm 0.02$ & $0.3 \pm 0.02$ \\
\hline
\end{tabular}

SBP, systolic blood pressure; DBP, diastolic blood pressure. ${ }^{\text {aP }}<0.05$ vs. Wild+ovx.

Fructose-feeding significantly induced the ovariectomyinduced increase in urinary deoxypyridinoline (Fig. 5C). These results suggest that fructose-fed insulin resistance directly accelerated estrogen deficiency-induced osteoporosis independent of blood pressure. Interestingly, the treatment of fluvastatin, but not pravastatin, significantly decreased osteoclast activity and improved bone mineral density, accompanied by the decrease in urinary deoxypiridinoline. In addition, fruvastatin decreased blood sugar induced by fructose feeding (fructose-fed: $144 \pm 5 \mathrm{mg} / \mathrm{dl}$; fructose-fed+ fluvastatin $132 \pm 5 \mathrm{mg} / \mathrm{dl}, \mathrm{P}<0.01)$.

\section{Discussion}

Recent therapy for diabetes, including improved glucose monitoring, insulin delivery methods, and pharmacologic treatments, are increasing the lifespan of patients, but increasing the risk of complications from extended exposure to diabetic conditions. Well known diabetic complications include retinopathy, neuropathy, and nephropathy. However, recently attention also focused on diabetic bone pathology. In the clinical view point, diabetic osteoporosis is mainly recognized as a significant comorbidity of type I diabetes mellitus (DM), but not type II DM $(1,2)$. In this study, we focused on bone pathology of insulin resistance, which could be involved in pre-hypertension and pre-diabetes. The 
A)
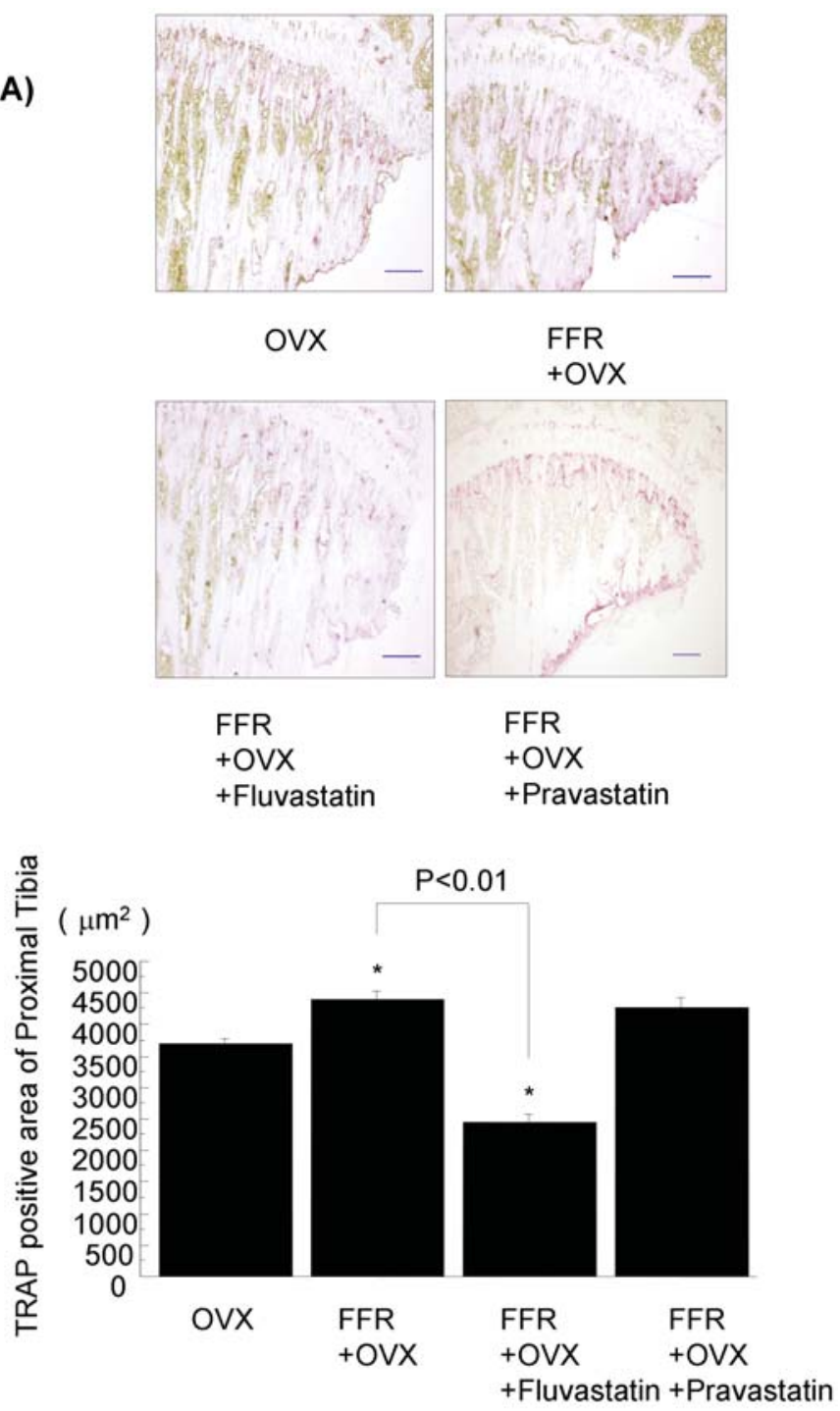

Figure 5. Effect of fluvastatin or pravastatin on Fructose-fed rats with ovariectomy. (A) TRAP staining of proximal tibia (Scale bar shows $300 \mu \mathrm{m}$ ), (B) bone mineral density by DEXA, and (C) urinary deoxypyridinoline after 28 days of each treatment. OVX, bilateral ovariectomy; FFR, fructose fed rat; Fluvastatin, treated with fluvastatin $(5 \mathrm{mg} / \mathrm{kg} /$ day $)$; Pravastatin, treated with pravastatin $(5 \mathrm{mg} / \mathrm{kg} /$ day $)$. Urinary deoxypyridinoline was adjusted for urinary creatinine concentration. ${ }^{*} \mathrm{P}<0.05$ vs. OVX; ${ }^{\dagger} \mathrm{P}<0.05$ vs. FFR + OV. $\mathrm{n}=6-10$ per group.

present study demonstrated that fructose-fed rat, one of the insulin resistance models with ovariectomy showed accelerated osteoporosis. Interestingly, fluvastatin attenuated the development of osteoporosis.

Simvastatin, mavastatin, fluvastatin, and lovastatin stimulate bone formation (22), and simvastatin and pitavastatin increase human osteoblast differentiation $(23,24)$. In addition to stimulating bone formation, statins may also inhibit resorption, in a similar way as described for some bisphosphonates $(25,26)$. Because statins also reduce the synthesis of other isoprenoid intermediates, such as farnesylpyrophosphate (FPP) and geranylgeranylpyrophosphate (GGPP), which are important lipid attachments for posttranslocational modification of the small GTP-binding protein Ras and Ras-like proteins (i.e. Rho and Rac). Nitrogencontaining bisphosphonates also act on the mevalonate path-

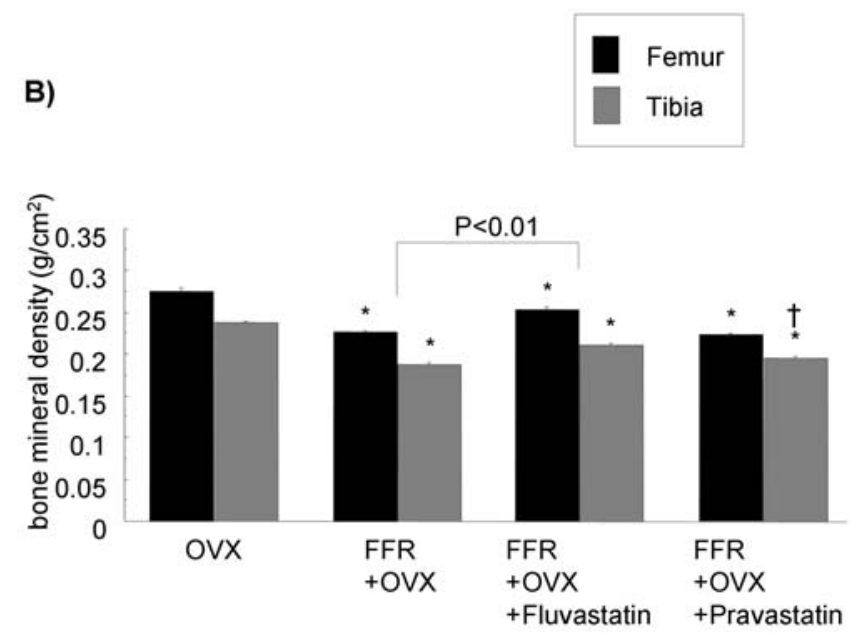

c)

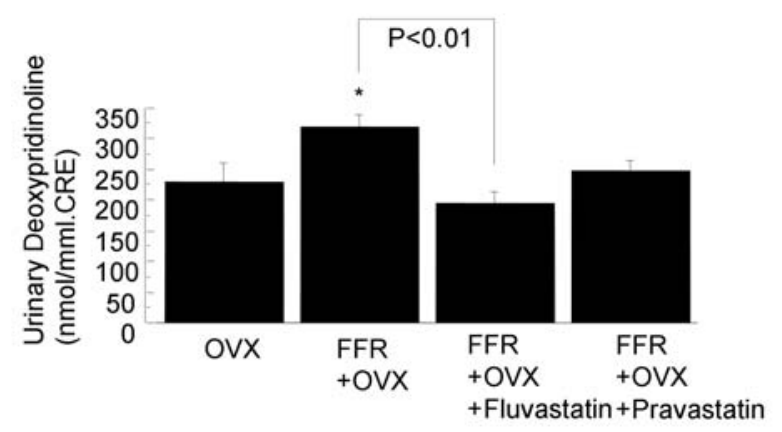

way to reduce the prenylation of GTP-binding proteins, which blocks osteoclast activity and inhibits osteoblast apoptosis $(25,26)$. However, pravastatin has different pharmacokinetics from other statins, with a large uptake by the liver via an active transport system, which limits its availability at other sites. This may explain the lack of effects seen in the analysis of the LIPID study (7), in which all the patients were taking pravastatin. Indeed, the present study demonstrated that fluvastatin, but not pravastatin, significantly decreased ROS production, and the antioxidant effect of fluvastatin is necessary to inhibit osteoclast activation and differentiation, in addition to the classical well-known mechanism through a reduction in prenylation of GTP-binding proteins.

Overall, the present study demonstrated potent inhibitory effects of fluvastatin on osteoclast activation and differentiation due to both an antioxidant effect and the reduction in prenylation of GTP-binding proteins. These favorable aspects of fluvastatin suggest the beneficial effects on osteoporosis and the reduction in bone fractures in human patients. Although the present study did not include a clinical evaluation, a previous study clearly demonstrated the superiority of fluvastatin compared to pravastatin for prevention of osteoporosis in patients (27). Furthermore, according to a pharmacokinetic study, the mean highest plasma concentration of fluvastatin after oral administration at a dose of $40 \mathrm{mg}$ /day for 6 days was almost $1 \mu \mathrm{M}$ (28), while the inhibitory effect of fluvastatin on osteoclast activation occurs at $100 \mathrm{nM}(1 / 10$ the plasma concentration at a therapeutic dosage) as shown in this study. Of importance, the effective concentration of fluvastatin in this study seems to be much lower than the concentration in human plasma. In contrast, although simvastatin also 
has inhibitory effects on osteoclast activation and differentiation, its effective dose (100 nM, data not shown) is much lower than the concentration in human plasma (the mean highest plasma concentration of simvastatin after oral administrations at a dose of $20 \mathrm{mg}$ /day for a period of 6 days was almost $17.8 \mathrm{nM}$ ). Further clinical studies comparing bone turnover, density, and risk of fracture among various statins in prospective trials are necessary. The present study demonstrates that fluvastatin significantly attenuated osteoclast differentiation and activation through blockade of the classical mevalonate pathway and an antioxidant action, leading to prevention of osteoporosis.

\section{Acknowledgments}

We thank Miss Natsuki Yasumasa for her technical assistance. Fluvastatin was kindly donated by Novartis Pharma. This work was partially supported by a Grant-in-Aid from the Organization for Pharmaceutical Safety and Research, a Grant-in-Aid from The Ministry of Public Health and Welfare, a Grant-in-Aid from Japan Promotion of Science, and through Special Coordination Funds of the Ministry of Education, Culture, Sports, Science and Technology, the Japanese Government.

\section{References}

1. Rosen CJ and Bouxsein ML: Mechanisms of disease: is osteoporosis the obesity of bone? Nat Clin Pract Rheumatol 2: 35-43, 2006.

2. McCabe LR: Understanding the pathology and mechanisms of type I diabetic bone loss. J Cell Biochem 102: 1343-1357, 2007.

3. Basaria S: Link between diabetes and osteoporosis. Diabetes Care 23: 564-565, 2000

4. Edwards CJ, Hart DJ and Spector TD: Oral statins and increased bone-mineral density in postmenopausal women. Lancet 355 : 2218-2219, 2000.

5. Chan KA, Andrade SE, Boles M, et al: Inhibitors of hydroxymethylglutaryl-coenzyme $A$ reductase and risk of fracture among older women. Lancet 355: 2185-2188, 2000.

6. Wang PS, Solomon DH, Mogun H and Avorn J: HMG-CoA reductase inhibitors and the risk of hip fractures in elderly patients. JAMA 283: 3211-3216, 2000

7. Reid IR, Hague W, Emberson J, et al: Effect of pravastatin on frequency of fracture in the LIPID study: secondary analysis of a randomised controlled trial. Long-term Intervention with Pravastatin in Ischaemic Disease. Lancet 357: 509-512, 2001.

8. Van Staa TP, Wegman S, de Vries F, Leufkens B and Cooper C: Use of statins and risk of fractures. JAMA 285: 1850-1855, 2001 .

9. Cappuccio FP, Kalaitzidis R, Duneclift S and Eastwood JB: Unravelling the links between calcium excretion, salt intake, hypertension, kidney stones and bone metabolism. J Nephrol 13: 169-177, 2000.

10. Resnick LM, Laragh JH, Sealey JE and Alderman MH: Divalent cations in essential hypertension. Relations between serum ionized calcium, magnesium, and plasma renin activity. N Engl J Med 309: 888-891, 1983.
11. Hwang IS, Ho H, Hoffman BB and Reaven GM: Fructoseinduced insulin resistance and hypertension in rats. Hypertension 10: 512-516, 1987.

12. Suzumura K, Yasuhara M, Tanaka K, Odawara A, Narita H and Suzuki T: An in vitro study of the hydroxyl radical scavenging property of fluvastatin, and $\mathrm{HMG}-\mathrm{CoA}$ reductase inhibitor. Chem Pharm Bull 47: 1010-1012, 1999.

13. Venken K, Boonen S, Van Herck E, et al: Bone and muscle protective potential of the prostate-sparing synthetic androgen 7alpha-methyl-19-nortestosterone: evidence from the aged orchidectomized male rat model. Bone 36: 663-670, 2005.

14. Shimizu H, Sakamoto M and Sakamoto S: Bone resorption by isolated osteoclasts in living versus devitalized bone: differences in mode and extent and the effects of human recombinant tissue inhibitor of metalloproteinases. J Bone Miner Res 5: 411-418, 1990.

15. Sundaresan M, Yu ZX, Ferrans VJ, Irani K and Finkel T: Requirement for generation of $\mathrm{H}_{2} \mathrm{O}_{2}$ for platelet-derived growth factor signal transduction. Science 270: 296-299, 1995.

16. Deng XF, Rokosh DG and Simpson PC: Autonomous and growth factor-induced hypertrophy in cultured neonatal mouse cardiac myocytes. Comparison with rat. Circ Res 87: 781-788, 2000.

17. Vanden Hoek TL, Becker LB, Shao Z, Li C and Schumacker PT: Reactive oxygen species released from mitochondria during brief hypoxia induce preconditioning in cardiomyocytes. J Biol Chem 273: 18092-18098, 1998.

18. Shimizu H, Nakagami H, Tsukamoto I, et al: NFkappaB decoy oligodeoxynucleotides ameliorates osteoporosis through inhibition of activation and differentiation of osteoclasts. Gene Ther 13: 933-941, 2006.

19. Kunugiza Y, Tomita T, Tomita N, Morishita R and Yoshikawa H: Inhibitory effect of ribbon-type NF-kappaB decoy oligodeoxynucleotides on osteoclast induction and activity in vitro and in vivo. Arthritis Res Ther 8: R103, 2006.

20. Laufs U and Liao JK: Targeting Rho in cardiovascular disease. Circ Res 87: 526-528, 2000

21. Shimizu H, Nakagami H, Osako MK, et al: Angiotensin II accelerates osteoporosis by activating osteoclasts. FASEB J 22: 2465-2475, 2008.

22. Mundy G, Garrett R, Harris S, et al: Stimulation of bone formation in vitro and in rodents by statins. Science 286: 1946$1949,1999$.

23. Maeda T, Matsunuma A, Kawane T and Horiuchi N: Simvastatin promotes osteoblast differentiation and mineralization in MC3T3-E1 cells. Biochem Biophy Res Commun 280: 874877,2001

24. Ohnaka K, Shimoda S, Nawata H, et al: Pitavastatin enhanced BMP-2 and osteocalcin expression by inhibition of Rho-associated kinase in human osteoblasts. Bioch Biophy Res Commun 287: 337-342, 2001.

25. Plotkin LI, Weinstein RS, Parfitt AM, Roberson PK, Manolagas SC and Bellido T: Prevention of osteocyte and osteoblast apoptosis by bisphosphonates and calcitonin. J Clin Invest 104: 1363-1374, 1999.

26. Fisher JE, Rogers MJ, Halasy JM, et al: Alendronate mechanism of action: geranylgeraniol, an intermediate in the mevalonate pathway, prevents inhibition of osteoclast formation, bone resorption, and kinase activation in vitro. Proc Natl Acade Sci USA 96: 133-138, 1999.

27. Watanabe S, Fukumoto S, Takeuchi Y, Fujita H, Nakano T and Fujita T: Effects of 1-year treatment with fluvastatin or pravastatin on bone. Am J Med 110: 584-587, 2001.

28. Tse FL, Jaffe JM and Troendle A: Pharmacokinetics of fluvastatin after single and multiple doses in normal volunteers. J Clin Pharmacol 32: 630-638, 1992. 